University of South Carolina

Scholar Commons

$12-1981$

\title{
Metrizability and the Frechet-Urysohn Property in Topological Groups
}

Peter J. Nyikos

University of South Carolina - Columbia, nyikos@math.sc.edu

Follow this and additional works at: https://scholarcommons.sc.edu/math_facpub

Part of the Mathematics Commons

\section{Publication Info}

Proceedings of the American Mathematical Society, Volume 83, Issue 4, 1981, pages 793-801. (C) 1981 by American Mathematical Society

This Article is brought to you by the Mathematics, Department of at Scholar Commons. It has been accepted for inclusion in Faculty Publications by an authorized administrator of Scholar Commons. For more information, please contact digres@mailbox.sc.edu. 


\title{
METRIZABILITY AND THE FRÉCHET-URYSOHN PROPERTY IN TOPOLOGICAL GROUPS ${ }^{1}$
}

\author{
PETER J. NYIKOS
}

\begin{abstract}
A question of Arhangel'skii, whether weakly first countable topological groups are metrizable, is answered in two ways: if the Hausdorff axiom is assumed, the answer is yes, but in general a weakly first countable topological group need not be pseudometrizable. The former result is obtained as a corollary of a more general sufficient condition for a sequential group to be Fréchet-Urysohn. A general necessary and sufficient condition for a sequential group to be FréchetUrysohn is given, and a number of questions are raised. Examples are given to show in what respect the theorems of the paper are the "best possible".
\end{abstract}

In his 1966 survey paper [1], A. V. Arhangel'skii defined the $g f$-axiom of countability and tossed out the question of whether every topological group which satisfies it is metrizable. Tacit in this question is the assumption of the Hausdorff $\left(T_{2}\right)$ separation axiom, since there are elementary examples of pseudometrizable topological groups that are not metrizable. If one assumes the $T_{2}$ axiom, the answer is fairly easily shown to be affirmative, as several people independently proved soon after Arhangel'skii's paper appeared. However, if one reinterprets the question, "Is every topological group satisfying the gf-axiom of countability pseudometrizable?" it turns out that a very familiar topological space is a counterexample. Ordinarily topologists do not concern themselves with non- $T_{2}$ spaces, but the counterexample has some relevance to functional analysis: it is the product of $\mathbf{R}$ with the indiscrete topology, with the locally convex direct sum of countably many copies of $\mathbf{R}$ with the usual topology. The main result of $\S 1$ is that this space is pseudosymmetrizable. In $\$ 2$, we prove a necessary and sufficient condition for a topological group to be Fréchet-Urysohn and use a formal strengthening of it to give a new proof that every $T_{2}$ topological group satisfying Arhangel'skii's axiom is metrizable. The final section gives examples to show some more ways in which the theorems of this paper are "the best possible".

1. Definitions and an example. The concepts we deal with in this paper are generalizations of first countability and metrizability.

Definition 1. A topological space $X$ is weakly first countable [Arhangel'skii: satisfies the $g f$-axiom of countability] if for each point $x \in X$ it is possible to assign

Received by the editors March 16, 1979 and, in revised form, February 17, 1981.

1980 Mathematics Subject Classification. Primary 54O55, 22A05, 54A05; Secondary 54A10, 54E99.

Key words and phrases. Topological group, weakly first countable, first countable, (pseudo-)symmetrizable, sequential, Fréchet-Urysohn.

${ }^{1}$ Research supported in part by NSF Grant MCS-8003004. $0002-9939 / 81 / 0000-0576 / \$ 03.25$ 
a sequence $(B(n, x))_{n=1}^{\infty}$ of subsets of $X$ containing $x$ in such a way that $B(n, x) \supset$ $B(n+1, x)$ and so that a set $U$ is open if, and only if, for each $x \in U$ there exists $n$ such that $B(n, x) \subset U$.

Additional information on weakly first countable spaces may be found in [11] (where they are called " $g$-first countable"), [6], and [12]. They include, of course, the first countable spaces, as well as the pseudosymmetrizable spaces, which are those weakly first countable spaces in which $B(n, x)$ can be defined so that $y \in B(n, x)$ if, and only if, $x \in B(n, y)$. A definition along more traditional lines runs:

Definition 2. A topological space is pseudosymmetrizable if it admits a distance function $d$ such that (i) for each $x$ and $y$ in $X, d(x, y)=d(y, x) \geqslant 0$ and $d(x, y)=0$ if $x=y$ and (ii) a set $F$ is closed if and only if $d(x, F)>0$ for every point $x \in F$. (As usual, $d(x, F)=\inf \{d(x, y): y \in F\}$.) A symmetrizable space is a pseudosymmetrizable space in which $d(x, y)=0$ only if $x=y$.

Of course, if we let $B(n, x)=\{y: d(x, y)<1 / n\}$ in a pseudosymmetrizable space, we satisfy the definition of a weakly first countable space. It is also trivial to show that every pseudometrizable space is pseudosymmetrizable, and is metrizable if, and only if, it is symmetrizable (because every symmetrizable space is $T_{1}$ ). There exist symmetrizable spaces which are not even $T_{2}$. A $T_{2}$, nonregular example is the "plus" topology on the plane (Example 4, §3).

The following well-known theorems, as well as the definition of a topological group, may be found in [8].

TheOREM A. A topological group is pseudometrizable if (and only if) it is first countable.

TheOREM B. A topological group is metrizable if (and only if) it is $T_{2}$ and first countable.

One might expect the generalizations of these theorems to weakly first countable spaces to stand or fall together, but in fact they do not. One indeed obtains a theorem if "weakly" is inserted before "first countable" in Theorem B, but not in Theorem A. The root of this anomaly seems to lie in the Lemma below, which features a very natural convergence criterion for which some separation axiom is necessary.

Definition 3. A subset $A$ of a topological space $X$ is sequentially closed if, whenever $y$ is the limit of a convergent sequence of points in $A$, then $y$ is in $A$. A space is sequential if every sequentially closed subset is closed. A space $X$ is Fréchet-Urysohn (also simply called Fréchet) if every point in the closure of a subset $A$ of $X$ is a limit of a sequence of points of $A$.

Given any subset $A$ of a topological space $(X, \mathcal{T})$, let $A^{\wedge}$ be the set of all limits of sequences in $A$. Then is a closure operator on the underlying set $X$. Those acquainted with closure spaces [4, especially Chapter III] will note that the space $(X, \widetilde{T})$ is sequential if, and only if, it is the topological space associated with the closure space $\left(X,{ }^{\wedge}\right)$, while $(X, \mathcal{T})$ is Fréchet-Urysohn if, and only if, it is the space $\left(X,{ }^{\wedge}\right)$. But we will not need this observation for any of what follows. 
We will let $N$ stand for the set of positive integers. Unless otherwise stated, a sequence will be understood to be a function with domain $N$. The following lemma will not be used until the next section, but it is instructive to look at Example 2 below with the proof in mind.

LEMMA 1. Every weakly first countable topological space is sequential. Given a sequence $\left(x_{n}\right)_{n=1}^{\infty}$ in a weakly first countable space, the following condition is sufficient for $x_{n} \rightarrow x$, and is necessary if the space is $T_{2}$ :

$$
\begin{aligned}
& \text { For each integer } n \in N \text { there exists } g(n) \in N \text { such that } \\
& x_{m} \in B(n, x) \text { whenever } m>g(n)
\end{aligned}
$$

where $B(n, x)$ is as in Definition 1.

Proof. The sufficiency of (D) is clear. Therefore, if $A$ is sequentially closed in the weakly first countable space $X$, there exists for each $y \notin A$ a "weak neighborhood" $B(n(y), y)$ missing $A$. Hence $X \backslash A$ is open. If $X$ is $T_{2}$, then the range of a convergent sequence is either a finite discrete closed subspace or else forms, together with its limit point, a closed subspace homeomorphic to $\omega+1$ with the order topology, with the nonisolated point as the limit of the sequence. Suppose then that $x_{n} \rightarrow x$, and that there exists $n$ such that, for infinitely many $m$, $x_{m} \notin B(n, x)$. Let $S$ be the range of a subsequence of such terms. Every point of $X \backslash(S \cup\{x\})$ has a neighborhood which misses $S$, and so each point of $X \backslash S$ has a weak neighborhood contained in $X \backslash S$, which is thus a neighborhood of $x$. This contradicts the claim $x_{n} \rightarrow x$.

The following example [though not all of its properties which we list] is well known from the theory of topological vector spaces [14, Chapter II, §6].

EXAMPLE 1. Let $E$ be the vector space of all sequences of real numbers with finite support. [The support of a sequence is its set of nonzero terms.] We give $E$ the box product topology. In other words, a base for $E$ is the collection of all sets of the form

$$
\Gamma_{n=1}^{\infty} U_{n}=\left\{x \in E: x_{n} \in U_{n} \text { for all } n\right\}
$$

where $U_{n}$ is an open set in the usual topology of $\mathbf{R}$ for all $n$. Thus $E$ is the locally convex direct sum of countably many copies of $R$ [14, p. 55], and can abstractly be characterized as "the" countable-dimensional (in the Hamel sense) vector space over $\mathbf{R}$ with the finest locally convex topology. Further information on this space may be found in [14, p. 69, Exercise 7] where it is stated that a convex subset of $E$ is closed if (and only if) its intersection with each finite-dimensional subspace is closed. The following proof that $E$ is sequential can easily be modified to show that "convex" is unnecessary in this exercise.

Suppose $A$ is a sequentially closed subset of $E$. Let $A_{n}$ (resp. $E_{n}$ ) be the set of all points of $A$ (resp. $E$ ) whose support is $\{k \in N: k \leqslant n\}$. Of course, $A=\cup_{n=1}^{\infty} A_{n}$, $E=\cup_{n=1}^{\infty} E_{n}$. To show that $A$ is closed, we may assume without loss of generality that $0 \notin A$, and exhibit a neighborhood of 0 that misses $A$.

Since $A$ is sequentially closed, there exists $\varepsilon_{1}>0$ such that $|f(1)|>\varepsilon_{1}$ for all $f \in A_{1}$. Let $U_{1}=\left(-\varepsilon_{1}, \varepsilon_{1}\right)$. Induction hypothesis: for each $i<n$ we have defined $\varepsilon_{i}$ 
such that for each $f \in A_{n-1}$, there exists $k<n$ such that $|f(k)|>\varepsilon_{k}$. Now suppose that for each $\varepsilon>0$ there exists $g_{\varepsilon} \in A_{n}$ such that $\left|g_{\varepsilon}(k)\right| \leqslant \varepsilon_{k}$ for all $k<n$, $\left|g_{\varepsilon}(n)\right|<\varepsilon$. Let $B=\left\{g_{1} / n: n \in N\right\}$. Then $B \subset E_{n}$, and in the natural isomorphism of $E_{n}$ with Euclidean $n$-space, $B$ has a bounded image, hence $B$ must have a sequence of distinct points converging to some $f \in E_{n}$. It is clear that any such $f$ is in $A_{n-1}$ (and, in particular, its $n$th coordinate must be 0 ) and $|f(k)| \leqslant \varepsilon_{k}$ for all $k<n$, contradiction. Hence we can pick $\varepsilon_{n}>0$ such that for each $f \in A_{n}$ there exists $k<n+1$ such that $|f(k)|>\varepsilon_{k}$.

With $\varepsilon_{n}$ chosen for each $n$, let $U=\left\{g:|g(n)|<\varepsilon_{n}\right.$ for all $\left.n\right\}$. Then $U$ is a neighborhood of 0 that misses $A$.

It is easy to see that $E$ is not first countable, or even Fréchet-Urysohn: the collection of functions $f_{n k}$ whose first coordinate is $1 / n$ and whose $n$th coordinate is $1 / k$ is easily seen to have 0 in its closure; but no sequence of these functions converges to 0 . An immediate consequence of Theorem 2 below is that $E$ is not weakly first countable.

Problem 1. The above proof can easily be modified to show that the direct sum of countably many locally compact metrizable groups with the box product topology is sequential. What happens if "locally compact" is omitted, or replaced by "complete"??

EXAMPLE 2. Let $G=\mathbf{R} \times E$ with $E$ as in Example 1, and give the extra copy of $\mathbf{R}$ the indiscrete topology. Obviously, $G$ is not pseudometrizable. Here is a sketch of the proof that $G$ is pseudosymmetrizable.

First we observe that if $f_{n} \rightarrow f$ in $E$ then there exists $m$ such that $\left\{f_{n}: n \in N\right\} \subset$ $E_{m}$. This is proven using the same techniques which showed $E$ to be sequential.

We now write the members of $G$ as functions from $\omega$ into $\mathbf{R}$. For $f, g \in G$ define $t(f, g) \leqslant r$ (where $r>0)$ to mean that $f(i)=g(i)$ for $i>|f(0)|+|g(0)|$ and $\mid f(i)-$ $g(i) \mid \leqslant r$ if $0<i \leqslant|f(0)|+|g(0)|$. Let $d(f, g)=1$ if $t(f, g) \leqslant r$ for any $r<1$, otherwise let

$$
d(f, g)=\inf \{r: t(f, g) \leqslant r\} .
$$

Clearly $d(f, g)=d(g, f) \geqslant 0$ for all $f, g \in G$.

Claim. If $A \subset G$, then $A$ is closed iff $d(f, A)>0$ for all $f \notin A$. The "only if" part is routine: if $A$ is closed and $f \notin A$, then there is a set of the form $\mathbf{R} \times \Gamma_{n=1}^{\infty} U_{n}$ missing $A$ and containing $f$.

So suppose that $A$ is nonempty and $d(f, A)>0$ for all $f \notin A$. Then $A=\mathbf{R} \times B$ for some $B \subset E$. It is enough to show that $B$ is sequentially closed in $E$. Suppose that $f_{n} \rightarrow f$ with $f_{n} \in B$ for all $n$, and pick $m$ such that $f_{n} \in E_{m}$ for all $n$. Let $k>m$, let $g \in G$ be the function satisfying $g(0)=k, g(i)=f(i)$ for all $i>0$, and let $g_{n}$ be any function in $G$ extending $f_{n}$. Then $g_{n} \in A$ for all $n$, and it is routine to verify that $d\left(g_{n}, g\right) \rightarrow 0$. Hence $g \in A$, and $f \in B$.

This proof is a modification of the author's proof that $G$ is weakly first countable. The definition of $d$ is due to Sheldon Davis.

\footnotetext{
${ }^{2}$ ADDED IN PROOF. E. K. van Douwen has shown that if even one factor is not locally compact, and infinitely many are not discrete, the resulting space is not sequential.
} 
Example 2 shows that pseudosymmetrizability is an exception to the rule of thumb that "generalized metric" properties are preserved by retracts. The obvious retraction of $G$ onto $\{0\} \times E$ is even an open map, and $G$ is pseudosymmetrizable, but $\{0\} \times E$ is not even weakly first countable.

2. Obtaining the Fréchet-Urysohn property. The main theorems of this section revolve around the question of when a sequential topological group is FréchetUrysohn. The first key theorem concerns the following condition, which is satisfied by any $T_{2}$ weakly first countable space:

Let $x \in X$ and for each positive integer $m$ let $\left(x_{n}^{m}\right)_{n=1}^{\infty}$ be a sequence convergent to $x$. It is possible to choose a term $x_{j(m)}^{m}$ for each $m$ in such a way that $\left(x_{j(m)}^{m}\right)_{m=1}^{\infty}$ converges to $x$.

Indeed, (*) is an immediate consequence of (D) in Lemma 1.

THEOREM 1. Let $G$ be a group with a topology $\mathcal{T}$ satisfying (*), such that the group multiplication is jointly continuous. If $(G, \mathcal{T})$ is sequential, then it is Fréchet-Urysohn.

Proof. Let $e$ denote the identity element of $G$. Suppose $G$ is not FréchetUrysohn. Then there is a subset $A$ of $G$ such that $A^{\mu}$ contains a point $x$ not in $A^{\wedge}$. By translating $A$ through multiplication by $x^{-1}$, we may assume $x=e$ without loss of generality.

Let $\left(x_{n}\right)_{n=1}^{\infty}$ be a sequence of points of $A^{\wedge}$ converging to $e$. For each $x_{n}$ let $\left(x_{n j}\right)_{j=1}^{\infty}$ be a sequence of points of $A$ converging to $x_{n}$. Since multiplication is separately continuous, $\left(x_{n}^{-1} x_{n j}\right)_{j=1}^{\infty}$ converges to $e$ for each $n$. By (*) it is possible to pick $j(n)$ for each $n$ so that $\left(x_{n}^{-1} x_{n j(n)}\right)_{n=1}^{\infty}$ converges to $e$. By joint continuity of multiplication, $\left(x_{n} x_{n}^{-1} x_{n j(n)}\right)_{n=1}^{\infty}=\left(x_{n j(n)}\right)_{n=1}^{\infty}$ also converges to $e$, contradicting the assumption that $e$ is not in $A^{\wedge}$.

As a corollary, we have

THEOREM 2. Let $G$ be a group with a weakly first countable topology with respect to which multiplication is jointly continuous. If $G$ is $T_{2}$, then $G$ is first countable.

Proof. By Lemma 1 and the discussion surrounding (*), $G$ satisfies the hypotheses of Theorem 1 . Therefore $G$ is Fréchet-Urysohn, and any weakly first countable Fréchet-Urysohn space is first countable [11].

The conclusion cannot be strengthened to " $G$ is metrizable" (see Example 3), but we do have

THEOREM 3. A topological group is metrizable if, and only if, it is $T_{2}$ and weakly first countable.

Proof. By Theorems A and 2.

The condition (*) cannot be eliminated from Theorem 1, as Example 1 above shows. However, it can be weakened (at least formally: see Problem 2). The proof of Theorem 1, with insignificant changes, shows that the following condition is also 
sufficient for a sequential group with jointly continuous multiplication to be Fréchet-Urysohn:

Let $x \in X$ and for each positive integer $m$ let $\left(x_{n}^{m}\right)_{n=1}^{\infty}$ be a sequence converging to $x$. It is possible to choose a sequence $(m(k))_{k=1}^{\infty}$ of distinct positive integers and a sequence $(j(k))_{k=1}^{\infty}$ of positive integers in such a way that $\left(x_{j(k)}^{m(k)}\right)_{k=1}^{\infty}$ converges to $x$.

Our second key theorem is

\section{THEOREM 4. Every Fréchet-Urysohn topological group satisfies (**).}

Proof. Let $e$ denote the identity of a topological group $G$; it is enough to show (**) for any collection $\left(x_{n}^{m}\right)_{n=1}^{\infty}$ of sequences converging to $e$. For each positive integer $k$, the sequence $\left(x_{k}^{1} x_{n}^{k}\right)_{n=1}^{\infty}$ converges to $x_{k}^{1}$, and so the union $A$ of the ranges of all these sequences has $e$ in its closure. By hypothesis there is a sequence $S$ in $A$ converging to $e$.

Case I. There is an infinite sequence $\left(x_{k}^{1}: k \in K\right)(K$ an infinite subset of $N)$ such that the closures of $\left\{x_{j}^{1}\right\}$ and $\left\{x_{i}^{1}\right\}$ are disjoint from each other, and from the closure of $\{e\}$. Then there is such an $S$ whose terms are contained in $\left\{x_{k}^{1} x_{n}^{k}: n \in\right.$ $N, k \in K\}$. Because $e$ and $x_{k}^{1}$ are contained in disjoint open sets if $k \in K, S$ can only meet each sequence in finitely many terms, hence must have a subsequence of the form $\left(x_{k(i)}^{1} x_{n(i)}^{k(i)}\right)_{i=1}^{\infty}$ converging to $e$, with $k(i) \neq k(j)$ when $i \neq j$. But $\left(\left(x_{k(i)}^{1}\right)^{-1}\right)_{i=1}^{\infty}$ converges to $e$, and thus

$$
\left(\left(x_{k(i)}^{1}\right)^{-1} x_{k(i)}^{1} x_{n(i)}^{k(i)}\right)_{i=1}^{\infty}=\left(x_{n(i)}^{k(i)}\right)_{i=1}^{\infty}
$$

converges to $e$, as desired.

Case II. The following is an equivalence relation in a topological group: $x \equiv y$ iff $x \in \operatorname{cl}\{y\}$. Hence in any case not covered by Case I, there are only finitely many equivalence classes of points in $\left(x_{k}^{1}\right)_{k=1}^{\infty}$. But then there exists $n$ such that all points of $\left(x_{k}^{1}\right)_{k=n}^{\infty}$ are in the equivalence class of $e$, because the sequence converges to $e$. Now if any of the other sequences $\left(x_{k}^{j}\right)_{k=1}^{\infty}$ is not of this degenerate form, then we renumber the sequences to make such a sequence be $\left(x_{k}^{1}\right)_{k=1}^{\infty}$ and argue as in Case I. If they are all of this degenerate form, the conclusion follows trivially.

Problem 2. The condition (**) looks annoyingly like (*). Are they equivalent for a topological group? Must every Fréchet-Urysohn topological group satisfy (*)? E. K. van Douwen has a construction [unpublished] of a countable regular topological space with one nonisolated point which satisfies (**) but not (*). The sequential coreflection [2], [7] of such a space is obviously Fréchet-Urysohn, and satisfies (**) but not (*) because the same sequences converge in both topologies. Since the coreflection topology is finer than the original, it will also be regular for a space with one nonisolated point.

Problem 3. Let $G$ be a group with a Fréchet-Urysohn topology with respect to which multiplication is jointly continuous. Does $G$ satisfy (*)? What about (**)?

Not every Fréchet-Urysohn space satisfies (**); for example, the sequential fan [13, Example D] does not satisfy it. 
Another interesting problem involves sequential order. Given a sequential space $X$, its sequential order $\sigma(X)$ is the smallest ordinal $\alpha$ such that $\alpha$ iterations of the operator " will give the closure of any subset of $X$. Thus $X$ is Fréchet-Urysohn if and only if $\sigma(X)=1$. There are examples of spaces satisfying $\sigma(X)=\omega_{1}$, the highest ordinal possible (see [2]). The Graev free topological group on $\omega+1$ is such an example [10], and it is possible to show that $\sigma(E)=\sigma(G)=\omega_{1}$ for Examples 1 and 2. In fact, one may ask:

Problem 4. If $X$ is a regular sequential semitopological group [8] which is not Fréchet-Urysohn, is $\sigma(X)=\omega_{1}$ ? What if $X$ is a topological group? weakly first countable?

An unpublished result of the author is that the following is a Hausdorff, symmetrizable, semitopological group $G$ with $\sigma(G)=2$ (hence Problem 3 has a negative answer if regularity is dropped): let $G$ be the collection of all finite and cofinite subsets of a countable set, the operation on $G$ being symmetric difference. A subset $A$ of $G$ is closed if, and only if, the union and intersection of every totally ordered subset of $A$ is in $A$. Leslie Foged [unpublished result] has also constructed regular, countable homogeneous spaces of every finite sequential order.

3. More examples. Examples 1 and 2 have already shown some ways in which Theorems 1 and 2 are "the best possible". Here are some more such examples.

EXAMPLE 3. In the group $S$ of real numbers with the Sorgenfrey topology (for which a base is the set of upper-half-open intervals), addition is jointly continuous and the space is first countable, but not metrizable. [ $S$ is not a topological group because inversion is not continuous.]

The next example shows that joint continuity in Theorems 1 and 2 cannot be replaced by separate continuity. In other words, it is not enough that the group be semitopological.

EXAMPLE 4. Let $G$ be the Euclidean plane with ordinary pointwise addition. Let a weak base at each point be formed by the collection of "plus signs" centered on it, with horizontal and vertical bars of length $1 / n$. What results is a Hausdorff semitopological group in which inversion is continuous but addition is not jointly continuous. And $G$ is not first countable, nor regular [6], but it is weakly first countable, indeed symmetrizable; given points $p$ and $q$, let $d(p, q)$ be the ordinary Euclidean distance if $p$ and $q$ are either on the same vertical or the same horizontal line; otherwise let $d(p, q)$ be 1 . This symmetric even has the property that every convergent sequence has a Cauchy subsequence, so that $G$ is a quotient $\pi$-image of a metric space [11].

It can be shown that the subgroup $H$ consisting of doubly rational points shares all the above properties with $G$ except nonregularity: $H$ is regular and countable, hence paracompact, but not first countable.

EXAMPLE 5. A countable topological group satisfying (*) nonvacuously which is not sequential. (Hence " $G$ is sequential" cannot be dropped from Theorem 1.) Let $G=\bigoplus\left\{G_{n m} \mid(m, n) \in N \times N\right\}$ where each $G_{m n}$ is a nontrivial cyclic group. [For example, if $G_{11}$ is of order 2 and every other summand is infinite, $G$ is isomorphic to the multiplicative group of nonzero rationals.] A base for the topology on $G$ is 
provided by the cosets of all the subgroups of the form $\bigoplus\left\{G_{m n} \mid(m, n) \in A\right\}$ where $A$ is a set which meets all but finitely many of the sets $\{m\} \times N$ and contains all but finitely many of the elements in each set which it meets. Then $G$ is a nondiscrete countable topological group in which there are no nontrivial convergent sequences. If one takes the topological group $G \times Q$, one obtains a nonmetrizable topological group in which each element is a limit of a sequence of distinct points, which satisfies (*) but is not sequential: the complement of $Q$ is dense but has no sequence converging to any member of $Q$.

Our last example shows that the conclusion of Theorem 1 cannot be strengthened to " $G$ is first countable".

EXAMPLE 6. Let $G$ be the direct sum of uncountably many nontrivial discrete abelian groups with the product topology. The elements of $G$ may be thought of as the members of the product with finite support (the support of an element being its set of nonzero coordinates). Thus a subset $A$ of $G-\{0\}$ has 0 in its closure if, and only if, for every finite set $B$ of coordinates, there is a member $a$ of $A$ whose support does not meet $B$. Since the supports are themselves finite, this is clearly equivalent to there being an infinite sequence of members of $A$ whose supports are disjoint. But any such sequence converges to 0 . Thus $G$ is Fréchet-Urysohn. Moreover, given any countably infinite collection of sequences converging to 0 , we can use induction to pick one element from each sequence so that the supports are disjoint, so $G$ satisfies (*). And, of course, $G$ is not first countable.

Interestingly enough, the following question seems to be unanswered:

Problem 5. Does there exist a countable Fréchet-Urysohn topological group which is not pseudometrizable? Does there exist a $T_{2}$ example?

ACKNOWLEDGEMENTS. The author wishes to thank Sheldon Davis for his help on Example 2, the referee for catching several errors in an earlier version of this paper, and the editor, D. J. Lutzer, for stopping this earlier version from going to press.

ADDED IN PROOF. G. Gruenhage has pointed out that the Fréchet-Urysohn property, together with $(* *)$, characterizes the countably bi-quotient spaces [E. Michael, A quintuple quotient quest, General Topology Appl. 2 (1972), 91-138] while the $w$-spaces [P. L. Sharma, Some characterizations of $W$-spaces and $w$-spaces, General Topology Appl. 9 (1978), 289-293] are precisely the Fréchet-Urysohn spaces which satisfy $(*)$.

\section{REFERENCES}

1. A. V. Arhangel'skii, Mappings and spaces, Russian Math. Surveys 21 (1966), 115, 162.

2. A. V. Arhangel'skii and S. P. Franklin, Ordinal invariants for topological spaces, Michigan Math. J. 15 (1968), 313-320.

3. E. Binz, Continuous convergence on $C(X)$, Lecture Notes in Math., vol. 469, Springer-Verlag, Berlin and New York, 1975.

4. E. Cech, Topological spaces, Interscience, New York, 1966.

5. S. W. Davis, G. Gruenhage, and P. J. Nyikos, $G_{\delta}$-sets in symmetrizable and related spaces, General Topology Appl. 9 (1978), 253-261.

6. S. P. Franklin and S. W. Williams, Nonregular separable spaces, Amer. Math. Monthly 75 (1968), 208.

7. H. Herrlich, Topologische reflexionen und coreflexionen, Lecture Notes in Math., vol. 78, SpringerVerlag, Berlin and New York, 1968. 
8. T. Husain, Introduction to topological groups, Saunders, Philadelphia, Pa., 1966.

$\rightarrow$ P. R. Meyer, Topologies of separate continuity on a cross space, Amer. Math. Monthly 75 (1968), 1128.

10. E. J. Ordman and B. V. S. Thomas, Sequential conditions and free topological groups, Report 77-7, Memphis State Univ., Dept. of Math. Sci., 1977.

11. F. Siwiec, On defining a space by a weak base, Pacific J. Math. 52 (1974), 233-245.

12. __ Generalizations of the first axiom of countability, Rocky Mountain J. Math. 5 (1975), 1-60.

$13 \longrightarrow$ Countable spaces having exactly one nonisolated point, I, Proc. Amer. Math. Soc. 57 (1976), 345-350.

14. H. H. Schaefer, Topological vector spaces; Macmillan, New York, 1966.

Department of Mathematics, University of South Carolina, Columbia, South Carolina 29210 